\title{
La santé bouge en Europe
}

De toute évidence les problèmes de la santé ont dépassé le stade de la stricte prérogative nationale au sein de l'UE. Trois axes de réflexions sont actuellement en cours.

D'abord la mobilité des patients et les services de santé. La Commission européenne a lancé une procédure de consultation à ce sujet avec date limite fixée au 31 janvier 2007. A ce propos se pose bien entendu non seulement la question des modalités d'assurance d'un pays à l'autre en dehors des situations d'urgence, mais surtout celle de la circulation des données. La carte européenne d'assuré est un premier pas. Pour l'heure elle ne fait que remplacer la formule E-111 avec les données administratives. Mais toute la question des données médicales et de façon générale de l'information électronique est en jeu. Le Comité permanent des médecins européens (CPME) y voue une attention toute particulière.

Dans ce contexte la sécurité du patient est également au centre des préoccupations. Sur ce thème, une déclaration commune des différentes organisations européennes en matière de santé (Professions de la santé, Consommateurs, CPME) faite à Luxembourg en juin 2005 avait été accueillie favorablement par la Direction générale SantéConsommation (DG-Sanco) de la Commission européenne. En fonction des réponses et des divers éléments rassemblés au cours de la procédure de consultation en cours, une ou plusieurs directives portant sur ces diverses questions seront élaborées.

Enfin un gentlemen's agreement concernant la libre circulation des médecins a été conclu à Edinbourg en 2005 pendant l'année de présidence européenne du Royaume
Uni. La présidence finlandaise a réuni les représentants des Etats membres à Helsinki le 23 octobre dernier pour en définir les modalités d'application. Cela concerne d'une part des qualifications professionnelles en fonction des règles établies par la directive 35/2005 CE sur la reconnaissance mutuelle des diplômes et d'autre part la situation des candidats à l'émigration sur le plan déontologique et judiciaire. Il est évident qu'il ne saurait être question d'accorder le droit de pratique à une consœur/confrère dans un autre pays que celui de son origine lorsque cette démarche vise à échapper à des sanctions de la part de son organisation professionnelle ou à des poursuites pénales.

Les accords bilatéraux ne sont pas une panacée. Certes la Suisse et la FMH sont associées dans une certaine mesure à ces discussions, en particulier par le biais du CPME dont nous sommes membres à part entière. Mais malheureusement nous ne pouvons participer aux décisions proprement dites, alors même que celles-ci devront être reprises dans le cadre de nos institutions, en particulier les instances mises en place par la Loi sur les professions médicales (LPméd) en ce qui concerne la reconnaissance des diplômes et de la formation acquises à l'étranger. La FMH suit donc avec beaucoup d'attention ces dossiers d'une importance capitale pour l'exercice de notre profession et l'évolution future du paysage sanitaire suisse.

A l'occasion de la séance ordinaire d'automne de la CPME, Luxembourg le 28 octobre 2006.

Dr Yves Guisan Vice-président FMH, Conseiller national 